Revista científica, arbitrada e indizada, bajo la modalidad electrónica.

Recibido: $15 / 07 / 2020$

Aceptado: 23/07/2020

\title{
PERSPECTIVAS DE LA INVESTIGACIÓN EN EL MUNDO: CASO PANAMÁ
}

Research perspectives in the world: Case Panama

\author{
Maricarmen Soto \\ Universidad Latina de Panamá/ \\ mcoromoto@ulatina.edu.pa
}

\section{RESUMEN}

Las ciencias de la vida, sociales, así como las humanas detectan problemas nuevos en el mundo. Por ello, los investigadores en un gran esfuerzo de aumentar la marca personal y las investigaciones en diversas líneas están dando respuesta a personas, organizaciones e innovando para lograr ese cometido en diversos países desde cada una de las especializaciones de la práctica multidisciplinaria, empoderando la institucionalización de protocolos, creando comités de bioéticas, haciendo cumplir las pautas éticas internacionales, además, de formar a estudiantes y tutores en los métodos para investigar. Panamá no escapa de esta búsqueda demostrando unos altos y bajos desde el año 2006 hasta la fecha, ubicándose hoy en 50\% de logros en investigación al corte del 2018. En un caso más específico la Universidad Latina, viene haciendo esfuerzos con programas de Coaching holístico para no solo enseñar los métodos, sino motivar creando interés en el proceso de investigación desde el Ser. En tal sentido, el objetivo planteado fue analizar las perspectivas de la investigación en Panamá, tomando una de sus universidades. Bajo un paradigma cualitativo, se realiza una aproximación hermenéutica sobre la propuesta de la Universidad Latina, con un diseño documental e informantes clave. Entre las evidencias obtenidas, esta la importancia de la acreditación del comité de bioética, consolidando las bases para los programas de fortalecimiento de investigadores que trabajen con seres humanos, revistas científicas, y visibilidad internacional. Dentro de las conclusiones se puede afirmar, que el proceso investigativo debe ser un eje trasversal, teórico-práctico, inmerso en actividades educativas, con el fin de desarrollar el pensamiento crítico, la publicación de manuscritos científicos en las revistas Conducta Científica y Gente Clave de ULatina, aumentar la visibilidad internacional en la búsqueda del ranking añorado por las universidades a partir de los productos intelectuales y la innovación conforme las necesidades investigativas.

Palabras clave: Perspectivas, Investigación, Universidad Latina de Panamá. 


\title{
Revista científica, arbitrada e indizada, bajo la modalidad electrónica.
}

\begin{abstract}
Life sciences, social as well as human sciences detect new problems in the world. Therefore, researchers in a great effort to increase personal branding and research in various lines are responding to people, organizations and innovating to achieve that task in different countries from each of the specializations of multidisciplinary practice, empowering institutionalization. of protocols, creating bioethics committees, enforcing international ethical guidelines, in addition, to train students and tutors in the methods to investigate. Panama does not escape this search by demonstrating highs and lows from 2006 to date, placing itself today in $50 \%$ of research achievements at the end of 2018 . Likewise, in a more specific case, Universidad Latina, has been making efforts with programs Holistic Coaching to not only teach the methods, but to motivate creating interest in the research process from the Self. In this sense, the objective was to analyze the perspectives of research in Panama taking one of its universities. Under a qualitative paradigm, a hermeneutical approach is made on the proposal of the Universidad Latina, with a documentary design and key informants. Among the evidence obtained, the accreditation importance of the bioethics committee was obtained, consolidating the bases for the strengthening programs of researchers working with human beings, scientific journals, and international visibility. Among the conclusions it can be affirmed that the research process must be a transverse, theoretical-practical axis, immersed in educational activities, in order to develop critical thinking, the publication of scientific manuscripts in the journals Scientific Conduct and Key People of ULatina, increase international visibility in the search for the ranking longed for by universities based on intellectual products and innovation according to research needs
\end{abstract}

Key words: Perspectives, Research, Universidad Latina de Panamá.

\section{INTRODUCCIÓN}

Para esta investigación se revisaron estadísticas, diversos paper sobre la investigación en América Latina, habiendo sido posible identificar desplazamientos teóricos-metodológicos que van apoyados en la visibilidad, en las especializaciones que aportan y otorga dirección a los estudios, definidos de acuerdo con las líneas de investigación. (Sunkel, 2006).

En vista del fortalecimiento de la investigación en el mundo, Panamá a través de su Secretaría Nacional de Ciencia y Tecnología SENACYT, (2018), imprime fuerza a través de la formación permanente a investigadores de las diversas Casas de Estudio, dando como 


\section{Revista científica, arbitrada e indizada, bajo la modalidad electrónica.}

resultado nuevas Revistas e indización de otras con el trabajo conjunto interuniversitario creando la evidencia en el Catálogo de Revistas de Panamá 2019.

Así es como en Panamá, se aumentan, se especializan, ademas optimizan hasta ser indexadas las revistas electrónicas, con base en la filosofía, licencia de acceso abierto, validez internacional y adquisición de índices, distribución, transformación, visualización.

\section{EL MÉTODO}

Para lograr este cometido se ha venido haciendo seguimiento a todos los aportes investigativos e inversión de la Secretaría Nacional de Ciencia y Tecnología SENACYT, a la cual se le ha aplicado un método hermenéutico, análisis de políticas, además de evidencias existentes que reflejan cómo es desarrollado el componente investigativo en el país. Bajo una complementariedad paradigmática, se desarrolló una ruta híbrida, (Azorín, 2012), donde se promueven mejores resultados con datos cuantitativos y cualitativos, con diseño documental, además fueron consultados expertos e informantes, para este manuscrito, con la necesidad de fortalecer la interacción entre el proceso docencia-servicioinvestigación. (Guanipa, 2013).

Entre los datos revisados e interpretados estuvo el aportado por Estrella (2019), quien afirmó en su ponencia en el Congreso Internacional de Investigación de la Universidad Americana, que desde el año 2006 hasta el corte realizado en el año 2018 se ha duplicado la inversión en materia de programas para apoyar la investigación en Panamá, por parte de la SENACYT. De unas cifras aproximadas de \$80mill en el 2006 hasta \$264mill para el 2018. Lo cual representan datos cuantitativos.

\section{RESULTADOS}

\section{La importancia del apoyo institucional del gobierno a la investigación en}

\section{Panamá}

La Secretaría Nacional de Ciencia y tecnología en Panamá, (SENACYT) (2018), viene recreando esfuerzos intensivos para crear vías apoyando la investigación. En tal 
sentido, el rol de la SENACYT ayuda a crear las rutas o procesos, invierte y financia la gestión del uniendo la experiencia de Institutos como el Gorgas, Investigaciones Científicas para el Desarrollo de Alta Tecnología (INDICASAT AIP), destacándose por la calidad en las investigaciones.

Además, SENACYT ha venido desarrollando una herramienta creada a través del Programa de Fortalecimiento de las Revistas Científicas de las Universidades de Panamá, como es la de orientar en la generación de revistas científicas y lograr que los docentes se motiven a investigar o plasmar sus investigaciones en formato científico en revistas creadas para ello.

Es por ello, que Panamá cuenta hoy en día con 37 revistas de corte científicoacadémico en formato digital: 33 de estas revistas son de universidades, 4 institucionales.

El Catálogo sirve como hoja de ruta para delinear el estado actual de las revistas, y para diseñar estrategias de fortalecimiento de las publicaciones científicas en Panamá.

Programa de Fortalecimiento de Revistas SENACYT

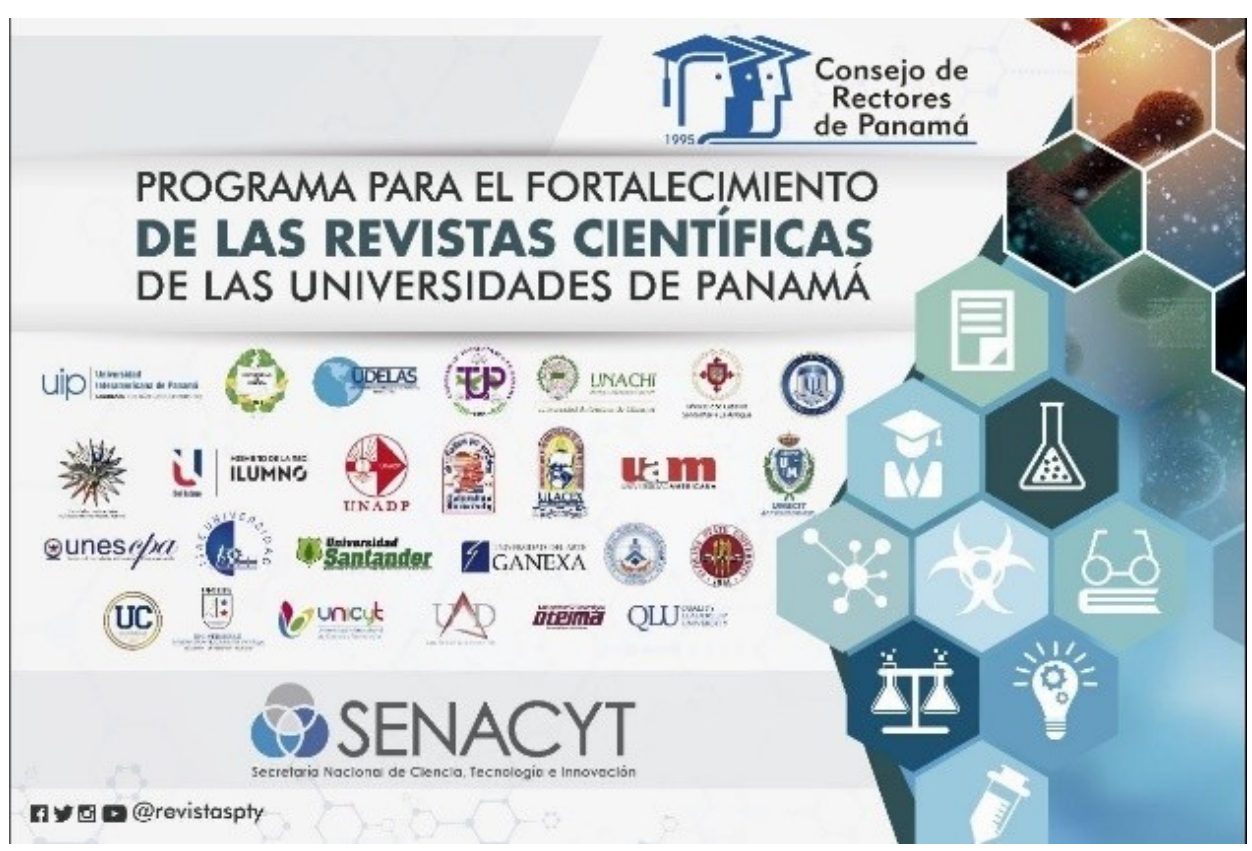

Figura 1 Fuente: SENACYT (2019) 
Catálogo de revistas de Panamá

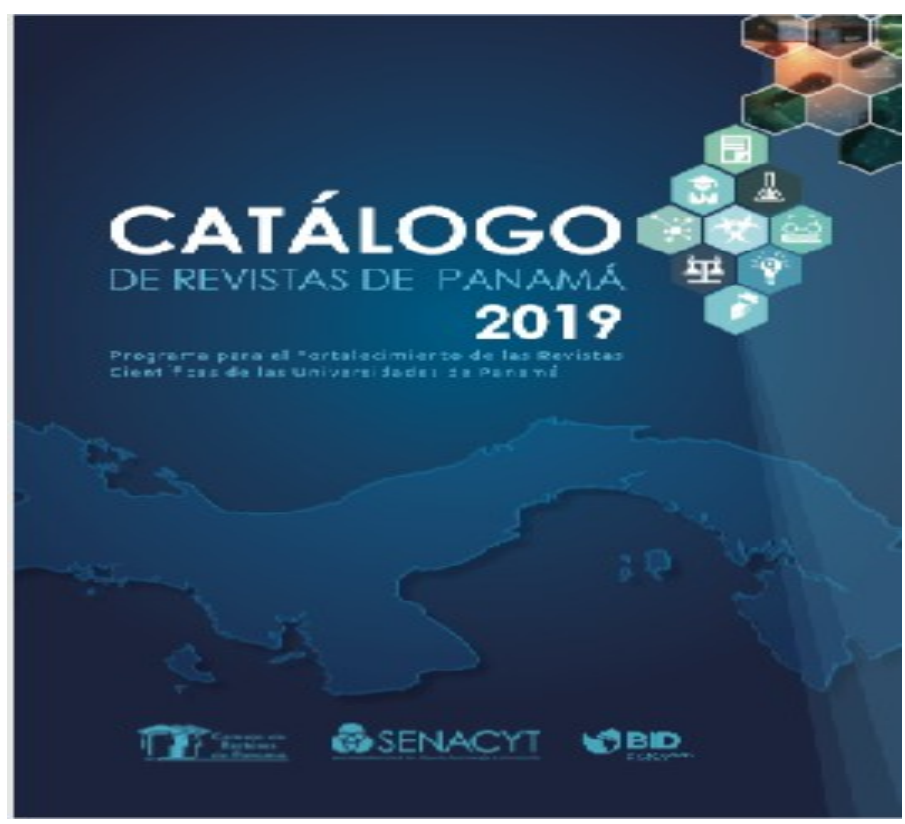

Figura 2 Fuente: SENACYT (2019)

Sin embargo, cabe destacar que las políticas públicas en Panamá, deben concienciar a todas las instituciones de educación superior para que investiguen y publiquen cada vez más, logrando profundizar la condición de academia, investigación y extensión. En este orden de ideas, la SENACYT, (2019), está consciente de ello y aumentado la inversión, pero sigue siendo poca. Los recursos económicos y humanos suelen ser escasos al observarse datos, de la misma SENACYT donde apenas un 0,23\% investigador, representa la inversión por cada 1000 habitantes en Panamá, siendo Panamá un país de aproximadamente 4.200.000 habitantes.

Por otro lado, Panamá, está en proceso de expansión significativo, cuenta con equipos de investigadores de alto performance, culturas y experiencias donde se mezclan saberes. A pesar de que hay debilidad en 2019 en el factor de impacto, existen revistas con indicadores bibliométricos basados en Google Scholar como: número de citaciones, número de publicaciones, h-index, visibilidad en directorios, catálogos y bases de datos internacionales. 
Cuadro comparativo de inversión países de la Región

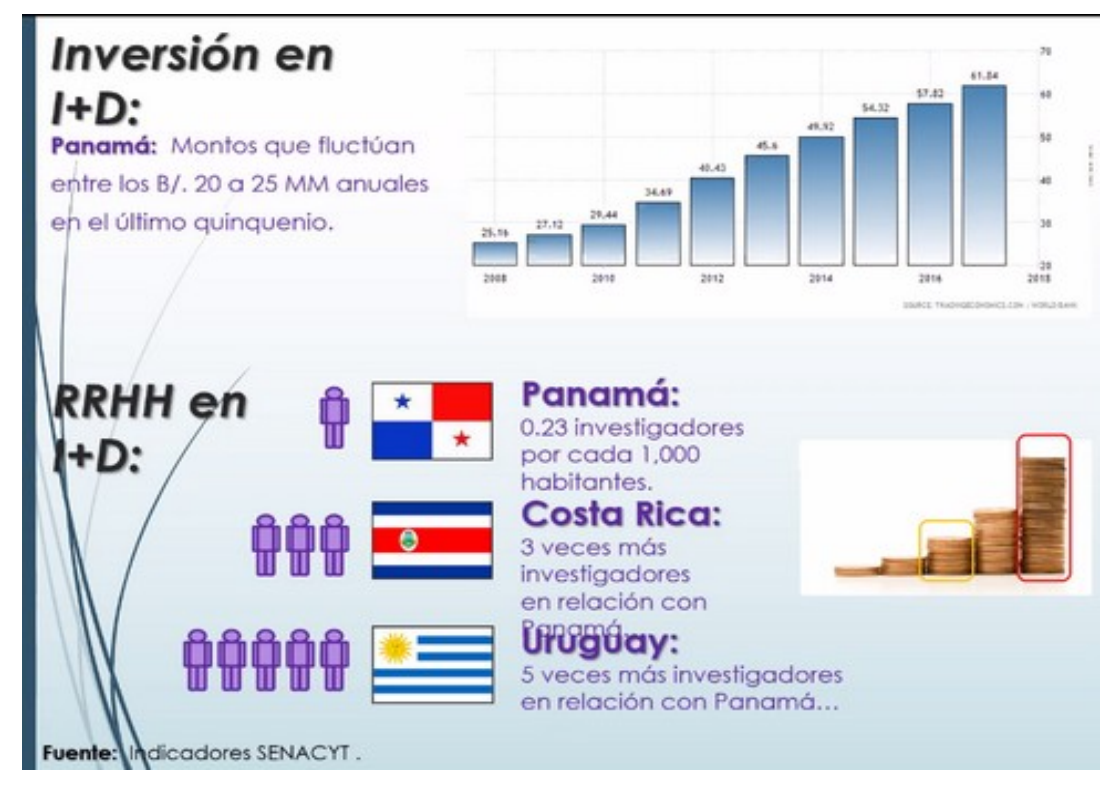

Gráfica 1 Fuente: SENACYT (2019)

Sin embargo, económicamente sigue siendo bajo el aporte del Estado para el impulso investigativo. Entonces ¿Cómo se avanza en la construcción de una academia generadora de pensamiento crítico, promovedora del diálogo con el Estado e instancias económicas, sin renunciar a su derecho a la generación de pensamiento liberador?, una pregunta vital que incluye en este proceso la investigadora Rodríguez (2012).

En este orden de ideas, consciente de la inversión y acceso a nuevas tecnologías en el mundo del conocimiento, Panamá, une esfuerzos en apoyo a la investigación con herramientas de enseñanza-aprendizaje en cada Casa de estudios. Por lo que sigue avanzando con el programa de fortalecimiento, el asesoramiento a la creación de repositorios institucionales, con miras a unificar en una sola base de datos el esfuerzo-país para el 2020, en cual tienen la visión primordial de cosechar en una gran base de datos denominada PRICILA, la cual será para febrero del próximo año que recoge todos los productos intelectuales nacionales, para la mayor visibilidad. 


\section{Revista científica, arbitrada e indizada, bajo la modalidad electrónica.}

Todo este gran proyecto en preparación formativa ameritará un arduo trabajo de los investigadores de cada universidad al tener que alimentar en los repositorios institucionales todas las comunidades investigativas al poder posteriormente alojarse en PRICILA.

El propósito final es proyectarse potenciando el desarrollo científico y tecnológico del país, logrando ser más sostenibles al cerrar la brecha de desigualdades, con la mejora de la calidad de vida, producto de la investigación de estudiantes, profesionales noveles, tutores e investigadores.

\section{Acreditación del comité de bioética de la Universidad Latina de Panamá}

En Panamá existe un Comité Nacional de Bioética (CNBI) (2018), el cual anualmente revisa, aprueba, aporta inversión a investigaciones en el ámbito de la salud que incluye al sector odontológico. Es por ello, que en mira de apoyar este proceso, la Universidad Latina de Panamá, se preparó para el requisito sine qua non de todas las investigaciones, prácticas profesionales, proyectos de investigación que tengan como sujetos de estudio, seres humanos.

En este orden de ideas, ya la Universidad cuenta con su Comité de Bioética (CBI) (2019), integrado por un personal certificado en buenas prácticas clínicas con aspectos necesarios a cubrir en la formación en esta materia, donde dicho ya acreditado formalmente por el Comité Nacional, puede ser centro de acopio, revisión no solo de sus propios protocolos de investigación sino de todas aquellas otras universidades e instituciones que lo soliciten.

Sobre el particular el CBI-ULatina es un grupo independiente, multidisciplinario de académicos colegiados en diferentes áreas de la salud, y científica. Su rol es salvaguardar la seguridad, dignidad, derechos y bienestar de todos los participantes de un estudio prevaleciendo la ética ante cualquier interés externo.

Con este logro se contribuye a la sociedad panameña y viene a ser un paso más para el fortalecimiento de la investigación y la innovación. Además, es una estrategia de filtro para la visibilidad internacional de la Universidad Latina y otras universidades que no 


\section{Revista científica, arbitrada e indizada, bajo la modalidad electrónica.}

poseen comité y requieras revisión de protocolos en las áreas de medicina, psicología, bioanálisis entre otras del área de la salud.

Procedimiento Proethos para la presentación y revisión de protocolos de investigación

En primera instancia cada estudiante o docente-investigador, de ensayos clínicos o aquellas investigaciones que involucre seres humanos, tiene que acceder a esta plataforma para registrar la investigación en proceso. En este sentido, se debe crear su cuenta para ingresar a dicha sitio web, una vez confirmada la cuenta, es ingresado el protocolo siguiendo los pasos señalados en el sitio, así ProEthos (2019), recibe el protocolo e inicia un proceso de revisión ética del documento.

La herramienta es una plataforma innovadora que optimiza la calidad, al revisar los elementos éticos de monitoreo a través de la rendición de cuentas, transparencia y eficiencia de los pasos de un protocolo de investigación, en los cuales participan sujetos humanos.

En un trabajo colaborativo ProEthos fue desarrollada por la Pontificia Católica Universidad de Paraná (Brasil), es un software de acceso gratuito para todos comités en cuatro idiomas (español, inglés, francés y portugués), es adaptable a las normas de cada institución y su implementación requiere de un mecanismo de gobernanza colaborativa y equipo de 7 países.

La plataforma ProEthos que pertenece a la Red de investigación de la Organización Mundial de la Salud y Organización Panamericana de la Salud. Es innovadora, al mejorar la calidad de los protocolos y monitorear que los mismos se ciñan a las normativas preestablecidas para las buenas prácticas clínicas, lo que significa que desde este momento la Universidad Latina de Panamá se apega a ProEthos. 
Logotipo del CBI de la ULatina
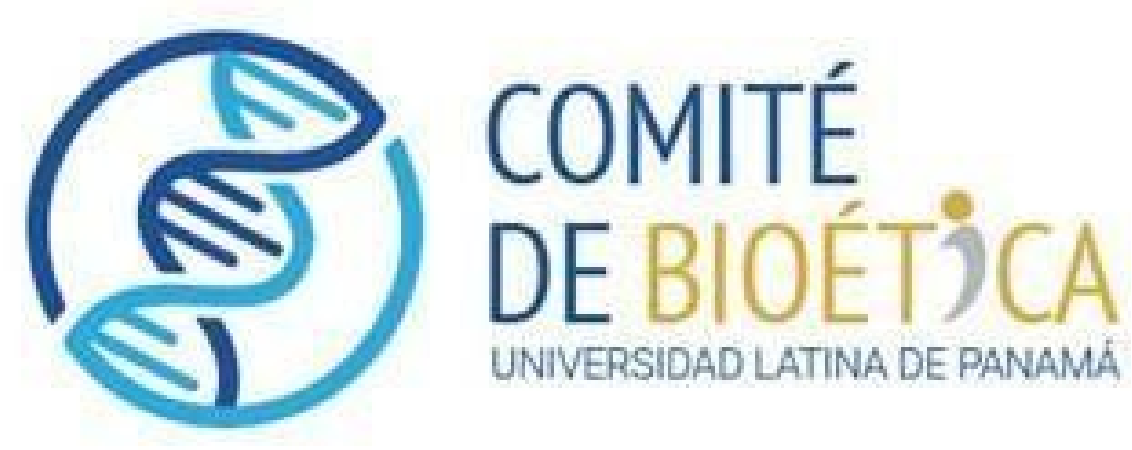

Figura 3 Fuente: Comité de Bioética de la ULatina (2019).

Perspectivas a partir de las evidencias de los programas de coaching holístico desarrollado por ULatina para investigadores y nóveles.

La actividad investigativa debe estar inserta en la vida cotidiana académica de la producción científica de la universidad, los procesos de acreditación la exigen, de hecho, la universidad posee un sistema propio denominado ACREULAT, producto de la investigación y propuesta de Morales (2018), para estandarizar la presentación de evidencias en la institución.

Ahora bien, lo concerniente a la formación de investigadores y la publicación de productos científicos en las revistas Conducta Científica y Gente Clave, propias de la ULatina, optimiza con prospectiva y perspectiva, como lo plantea Baldión (2019), por ello, es tan necesario la creación de grupos de investigación con estudiantes, para aportar al mundo la democratización de ideas innovadoras con proyectos entre pares, que contrasta pensamientos y enriquecen la vida académica. (Vega-Mosalve, 2019).

Las líneas de investigación, de la ULatina poseen una serie de componentes:

1. Revisión de las líneas de investigación

2. Potencialidades del currículo a nivel de grado y posgrado de acuerdo con lo aprobado por la Comisión Técnica de Acreditación (CTDA).

3. Talleres sobre el contenido y justificación de las líneas.

4. Compromisos con el mejoramiento continuo del proceso formativo de la carrera.

5. Actualización de las líneas

6. Creación de revistas electrónicas 


\section{Revista científica, arbitrada e indizada, bajo la modalidad electrónica.}

Líneas de Investigación potenciales de la ULatina

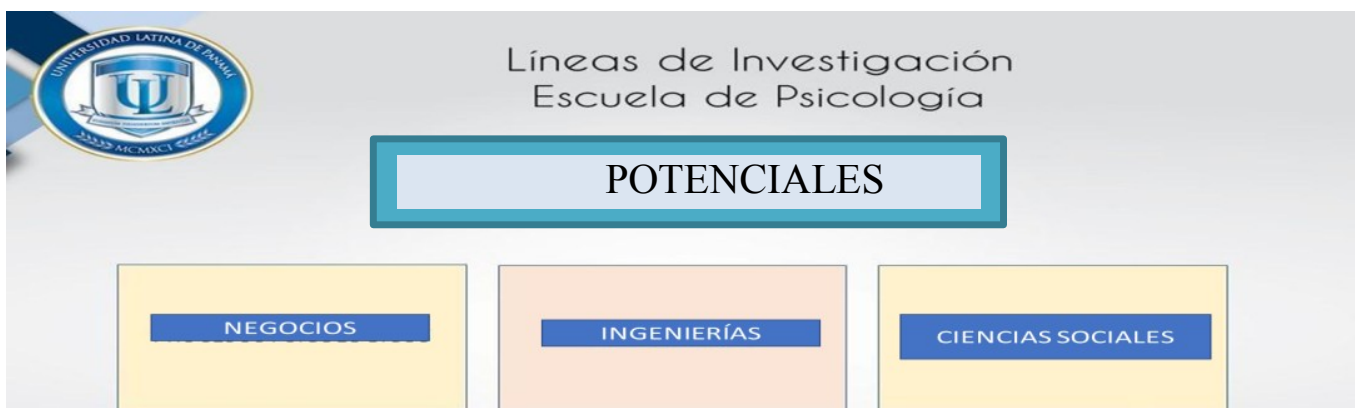

Figura 4. Fuente: Universidad Latina de Panamá, (2019).

Portal de revistas ULatina

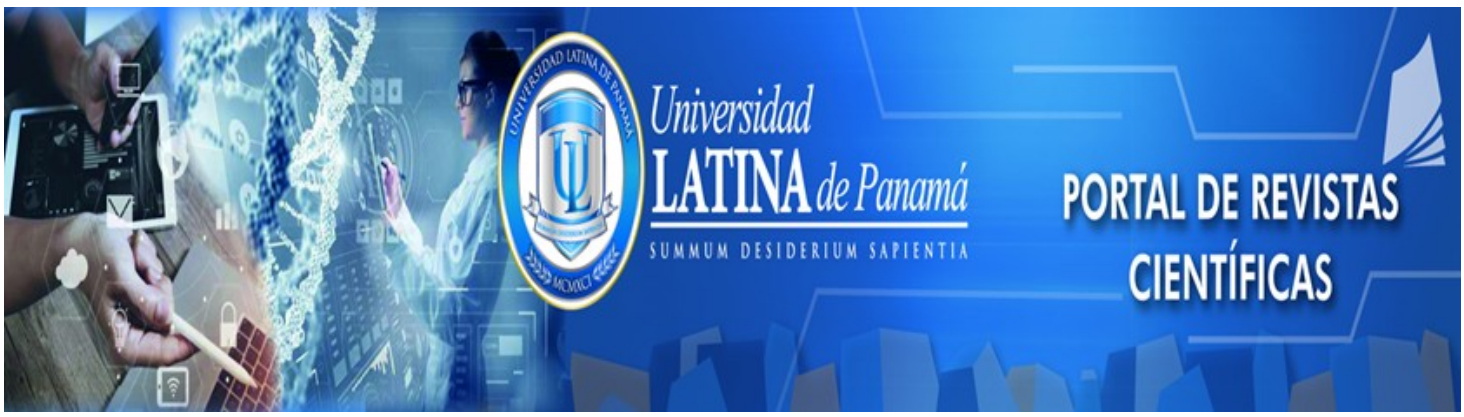

Figura 5. Fuente http://revistas.ulatina.edu.pa. (2019)

La figura 4, muestra las líneas de investigación vigentes hasta ese momento. En la misma se observa la necesidad de replantear o ampliarlas de acuerdo con modelos contextualizados con la realidad, para poder generar un modelo de líneas de investigación más adaptadas a las nuevas exigencias del país y del mundo, con óptica 5.0.

Una vez organizada la estructura conceptual investigativa tomando en cuenta lo tecnológico se alimentarán las líneas de investigación dentro del proceso docenciainvestigación, necesitando para ello, los siguientes recursos:

1. Humanos

a. Administrativos

b. Docentes

c. Estudiantes 


\section{Revista científica, arbitrada e indizada, bajo la modalidad electrónica.}

d. Equipos de trabajo

2. Técnicos y tecnológicos

a. Bibliotecas físicas y virtuales (inserción en el Repositorio PRICILA del país)

1. Docentes-investigadores: sistematización de la producción académica que incluya resultados de investigación en el proceso formativo, que incluye desarrollo de actividades investigativas: día científico con la participación de expositores nacionales e internacionales. (Numa-Sanjuan y Márquez, 2019).

2. Jornadas de actualización, simposios, poster, participación en otros eventos científicos.

3. Participación en eventos nacionales e internacionales.

4. Divulgación de la actualización de las líneas de investigación.

5. Crear las bases para la presentación de la revista electrónica de la carrera liderada por los estudiantes con la asesoría de docentes.

\section{Origen de la producción intelectual de la ULatina}

De acuerdo a lo expresado por Soto (2019), la universidad posee seis institutos de investigación que soportan a la dirección de investigación con sus producciones desde las aulas de clases, y los estudiantes en formación investigativa, conocidos como semilleros también, que viene a ser un investigador novel y justamente nace de esos espacios o grupos de investigación de los institutos, allí existe la producción y aprendizaje, que se relaciona teóricamente con un ambiente práctico a través con procesos sistemáticos de enseñanzaaprendizaje, además, las competencias en investigación para la formación de los investigadores de un mayor número de noveles. (Kozlowski, Ilgen,2006)

Al respecto, Álvarez (2019), expone que existen soportes epistemológicos para la construcción y reconstrucción de objetos de estudio en investigaciones ya sean en el sector salud o en las ciencias sociales y/o humanas y que se necesitan para formar a los investigadores noveles en la reconstrucción de problemáticas para lograr las evidencias, soluciones y/o propuestas. 
Estructura investigativa ULatina

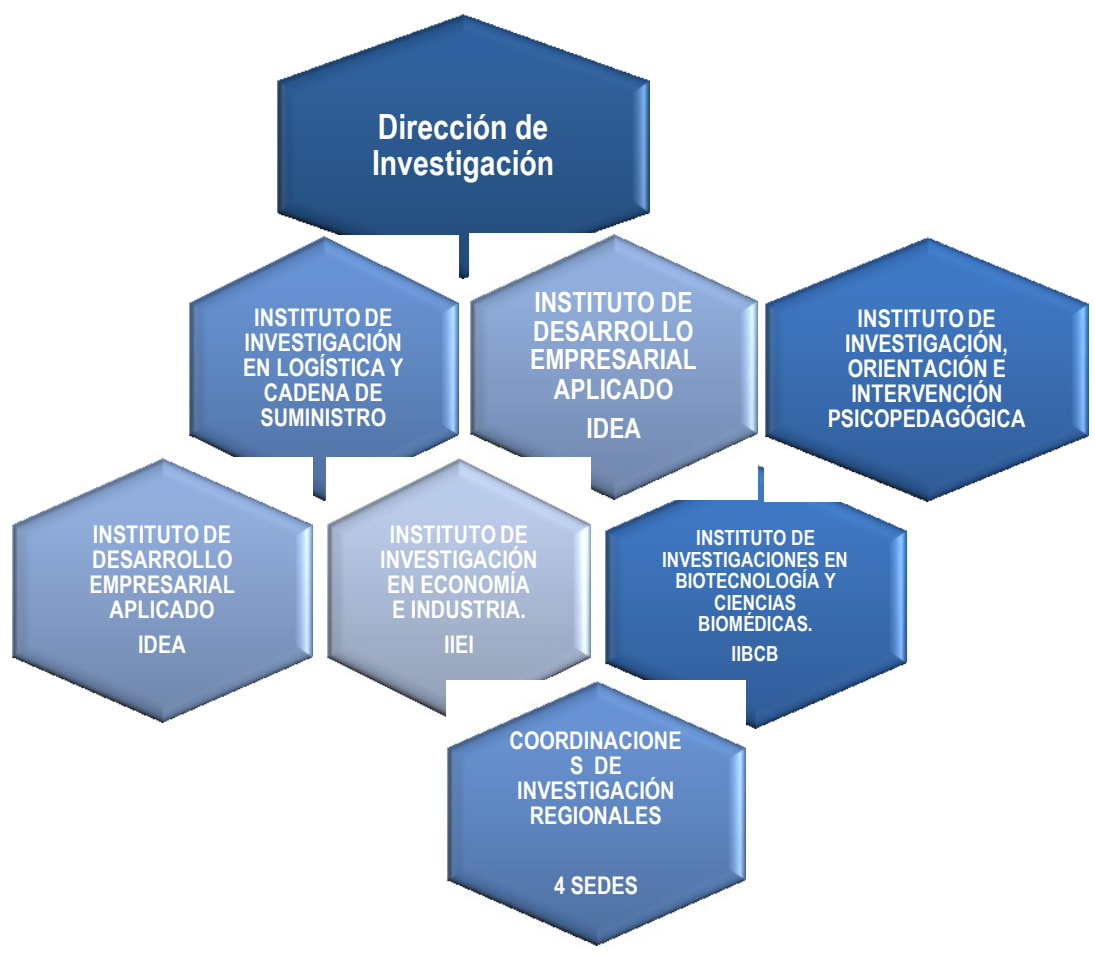

Figura 6. Fuente: ULatina. Dirección de Investigación (2019)

\section{CONCLUSIONES}

Una vez revisados todos los datos cuantitativos y cualitativos sobre la temática a nivel mundial y en Panamá, con una delimitación geográfica en la Universidad Latina, se obtuvo que pueden ser fortalecidas a través de mayores grupos de investigación las distintas Escuelas, motivando al estudiantado a investigar, reforzando el pensamiento crítico a través del programa de coaching holística para formación de investigadores que viene desarrollando la Dirección de Investigación, con el propósito de escribir y publicar un mayor números de artículos inéditos, proyectos e innovaciones. (Leyva, Gaitán, 2019).

Ademas se deben actualizar y fortalecer las líneas de investigación potenciales, creando un equipo multidisplinario, que trabaje permanentemente, como equipo en cada sub-línea tomando en cuenta las políticas de Estado, escenarios de incertidumbre, 


\section{Revista científica, arbitrada e indizada, bajo la modalidad electrónica.}

circunstancias de la Región que requieran, propuestas de acción e innovación diferenciales diferentes con alcances que soluciones temáticas y tópicos de interés.

Al final de todos estos procesos, las líneas matrices, las potenciales, así como las virtuales conformarán una suerte de entramado en espiral, con puntos de contacto entre los tres niveles; será un gran mapa que desplegará las posibilidades de una línea de investigación donde la Dirección de Investigación de Ulatina, tiene un papel de puente y coordinador primordial.

Otra herramienta de la que se hace uso hoy en día para la visibilidad nacional e internacional lo representan las revistas electrónicas, como los repositorios institucionales, en Panamá, de 5 repositorios apenas 3 son visibles. Por ello el proyecto PRICILA se esmera y durante este año se ha esperado su lanzamiento, para darle mayor visibilidad a las investigaciones del país.

Esta es la forma de potenciar y motivar la investigación, suelen ser unas herramientas valiosas, la ruta donde se pueda publicar todos los esfuerzos realizados por los grupos de investigación como proyectos y papeles de trabajo, que garanticen una calidad investigativa y una institución universitaria con pasos cuánticos hacia el futuro académico. (Numa-Sanjuan, Márquez, 2019).

\section{REFERENCIA BIBIOGRÁFICAS}

Álvarez, G. (2019). Construcción y reconstrucción del objeto de estudio en la investigación educativa. Revista Actualidades investigativas en educación. Vol 19 No. 3. Universidad de Costa Rica. Costa Rica.

Azorín, J. (2012). Métodos híbridos de investigación y dirección de empresas, ventajas e implicaciones. Dialnet. España.

Baldión, P. (2019). Situación y perspectiva de la investigación en las facultades de odontología de Colombia: Una reflexión. Artículos de opinión. Acta odontológica colombiana. Colombia.

Comité Nacional de Bioética de Panamá. (2018). Plantilla de trabajo. file://C:/Users//Downloads/Informe-Anual-2018-CEI-CSS.pdf consultada el 20desept19.

Comité de Bioética de la universidad Latina (2019). CBI Panamá. 


\section{Revista científica, arbitrada e indizada, bajo la modalidad electrónica.}

Estrella, J. (2019. Investigación en Panamá. Ponencia realizada en el I Congreso Internacional de Investigación de la UAM. Universidad del Caribe. Panamá

Guanipa, M. (2013). Investigación cuantitativa y cualitativa: interdependencia del método. Redalyc Revista REDHECS. Universidad Dr. Rafael Belloso Chacín. Venezuela.

Kozlowski, SWJ., Ilgen, DR (2006). Enhancing the Effectiveness of Work Groups and Teams. Michigan State University. EEUU.

Leyva, E., Gaitán, L. (2019). La importancia de las revistas científicas. Una nueva era para la revista odontológica mexicana. Vol 3 No. 1 UNAM. México.

Numa-Sanjuan, N., Márquez, R. (2019). Los Semilleros como espacios de investigación para el investigador novel. Propósitos y Representaciones, 7(1), 230-248. Doi: http://dx.doi.org/10.20511/pyr2019.v7n1.289

Morales, Ma. E. (2019). ACREULAT Sistema de acreditación de la Universidad Latina de Panamá. Panamá.

Portal de revistas de la Universidad Latina de Panamá. (2019).

ProEthos. De Neil, M. (2019). https://es.slideshare.net/ComunidadRedDes/la-plataforma proethos-para-comits de-tica-en-investigacin

Rodríguez, M. (2012). Líneas de investigación y dialogismo en los procesos investigativos en el campo universitario ecuatoriano. Latinoamérica. Revista de estudios Latinoamericanos. No. 54. Perú

Secretaría Nacional de Ciencia y Tecnología. SENACYT. (2019). Programa de creación de repositorios institucionales. Panamá.

SENACYT (2018). SENACYT, programa de Fortalecimiento de Revistas. Panamá.

Sunkel, G. (2006). El consumo cultural en América Latina. Construcción teórica y líneas de investigación. Colección Agenda Iberoamericana. Colombia.

Soto, M. (2019). Ponencia perspectivas de la investigación a nivel mundial Caso Universidad Latina de Panamá. Estructura departamental de la Dirección de Investigación. I Congreso Virtual de Investigación de la Florida Global University. Panamá

Vega-Mosalve, N. (2019). Estrategias de conformación y consolidación de semilleros de investigación en pregrado. Estudio de caso en una institución de educación superior en Colombia. Scielo. Vol. 10, No. 27. Colombia. 